\title{
Criopreservación de semen canino (Canis familiaris) en pajilla francesa en el municipio de Medellín, Antioquia
}

\author{
Canine semen (Canis familiaris) cryopreservation on french straw in \\ Medellín, Antioquia
}

\begin{abstract}
Arango-Múnera JD, Castrillón-Zuluaga V, Correa-Rendón N, Suarez-Delgado M, Carrillo-Gonzalez DF. Criopreservación de semen canino (Canis familiaris) en pajilla francesa en el municipio de Medellín, Antioquia. Rev Colombiana Cienc Anim. Recia. 2020; 12(1):e754. DOI: https://doi. org/10.24188/recia.v12.n1.2020.754
\end{abstract}

Universidad de Sucre, Colombia

Los autores permiten a RECIA reimprimir el material publicado en él. En caso de que un autor quiera traducir o usar una publicación parcial o completa de nuestro Diario, el autor debe obtener un permiso por escrito del editor de la revista.

Copyright (C) 2020. El (los) autor (es), Revista Colombiana de Ciencia Animal - RECIA. 2020. Este es un artículo de acceso abierto distribuido bajo los términos de Creative Commons Attribution 4.0 (https://creativecommons.org/licenses/by-nc-sa/4.0/), El uso, distribución o reproducción está permitido, siempre que se acrediten al autor original y al propietario del copyright y que se cite la publicación original en esta revista, de acuerdo con la práctica académica aceptada. No se permite el uso, distribución o reproducción que no cumpla con estos términos.

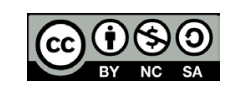




\title{
Criopreservación de semen canino (Canis familiaris) en pajilla francesa en el municipio de Medellín, Antioquia
}

\author{
Canine semen (Canisfamiliaris) cryopreservation on french straw in Medellín, Antioquia
}

Julián D. Arango Múnera. MV.

Universidad de Antioquia. Facultad de Ciencias Agrarias, Escuela DOI: https://doi.org/10.24188/recia.v12.n1.2020.754 de Medicina Veterinaria, Medellín, Colombia. jdamrn@hotmail.com

(iD http://orcid.org/0000-0003-3362-0508

\section{Vanessa Castrillón Zuluaga. MV.}

Universidad de Antioquia. Facultad de Ciencias Agrarias, Escuela de Medicina Veterinaria, Medellín, Colombia.

vanecazu@hotmail.com

(iD) http://orcid.org/0000-0002-9024-5196

Natalí Correa Rendón. MV.

Universidad de Antioquia. Facultad de Ciencias Agrarias, Escuela de Medicina Veterinaria, Medellín, Colombia.

natali.correa@udea.edu.co

(D) http://orcid.org/0000-0003-0510-3837

\section{Marcela Suarez Delgado, MV.}

Universidad de Antioquia. Facultad de Ciencias Agrarias, Escuela de Medicina Veterinaria, Grupo de investigación CENTAURO, Medellín, Colombia.

marcela.suarezdelgado@gmail.com

(D) http://orcid.org/0000-0003-0938-6897

Diego F. Carrillo-Gonzalez, ${ }^{*}$ Ph.D.

Universidad de Antioquia. Facultad de Ciencias Agrarias, Escuela de Medicina Veterinaria, One Health and Veterinary Innovative Research \& Development Group, Medellín, Colombia.

Universidad de Sucre, Facultad de Ciencias Agropecuarias, Departamento de Zootecnia, Sincelejo, Colombia

Recepción: 15 agosto 2019 fernando.carrillo@udea.edu.co

(i) https://orcid.org/0000-0002-0326-0815

Aprobación: 30 marzo 2020

Publicación: 30 abril 2020

\section{RESUMEN}

El objetivo de este trabajo fue aplicar la metodología de criopreservación de semen canino en pajilla francesa en el municipio de Medellín- Antioquia. Se realizó una colecta seminal a ocho caninos adultos, por método de mano enguantada, cada eyaculado fue evaluado macroscópica y microscópicamente y posteriormente diluido con Triladyl ${ }^{\circledR}$ a una concentración final de $80 \times 10^{6}$ espermatozoides/ml. Se empacaron en pajillas de 0,5 ml y fueron sometidas a criopreservación con un descenso de temperatura rápido, sometiendo las pajillas a vapores de nitrógeno durante 25 minutos y luego transferidas directamente a nitrógeno líquido (método de pajilla francesa). En el semen fresco, se observó una apariencia blanquecina lechosa, un volumen de $2.27 \mathrm{ml}( \pm 1.40)$ una concentración de $388.5 \times 10^{6}$ espermatozoides/ mL $( \pm 228.069)$, una motilidad individual de $79 \%( \pm 4 \%)$ y un vigor de $3.96( \pm 0.327)$. Se obtuvieron 123 pajillas, de las cuales se tomaron 
27 aleatoriamente y se les evaluó la motilidad individual $(51 \pm 19 \%)$ y vigor $(2.89 \pm 1.02)$. En conclusión, la aplicación

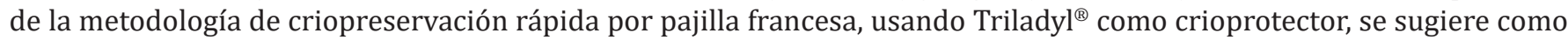
protocolo eficiente para la criopreservación de semen canino en el municipio de Medellín.

Palabras claves: Biotecnología, congelación, perro, reproducción.

\begin{abstract}
The objective of this work was to apply the canine semen cryopreservation methodology in French straw in MedellínAntioquia. A seminal collection was carried out to eight adult canines, by gloved hand method, each ejaculate was assessed macroscopically and microscopically and subsequently diluted with Triladyl ${ }^{\circledR}$ with a final concentration of $80 \times 10^{6} \mathrm{sperm} /$ $\mathrm{ml}$. Semen samples were packed in $0.5 \mathrm{ml}$ straws and a quick-freezing process was performed (nitrogen vapors for 25 minutes and then transferred directly to liquid nitrogen, french straw method). In fresh semen, a milky whitish appearance, a volume of $2.27 \mathrm{ml}( \pm 1.40)$, a concentration of $388.5 \times 10^{6} \mathrm{sperm} / \mathrm{mL}( \pm 228.069)$, individual motility of $79 \%( \pm 4 \%)$ and vigor of $3.96( \pm 0.327)$ were observed. 123 straws were obtained, of which 27 were taken randomly and individual motility $(51 \pm 19 \%)$ and vigor $(2.89 \pm 1.02)$ were evaluated. In conclusion, the application of the methodology of quickfreezing by french straw, using Trilady ${ }^{\circledR}$ as a cryoprotectant, is suggested as an efficient protocol for cryopreservation of canine semen in Medellín-Antioquia.
\end{abstract}

Keywords: Biotechnology, dog, quick-freezing, reproduction.

\title{
INTRODUCCIÓN
}

Diferentes técnicas biotecnológicas de reproducción animal han sido empleadas alrededor del mundo por parte de especialistas en reproducción, veterinarios, personas del común y criadores de perros (1), conduciendo así al conocimiento de las diferentes ventajas de estos procedimientos. La criopreservación de semen canino en particular, es una técnica que permite la prevención de enfermedades venéreas, la reproducción de individuos geográficamente aislados o de animales que por causas anatómicas o comportamentales no pueden llevar a cabo el proceso de monta natural. Adicionalmente, la criopreservación de semen, permite el uso pajillas provenientes de reproductores que han finalizado su período útil como sementales o que incluso, para la fecha de uso de la pajilla, ya se encuentran muertos (2). Del mismo modo, el proceso de criopreservación de semen canino, favorece la optimización del uso de semen de buena calidad, permitiendo usar un mismo eyaculado para inseminar a varias hembras, incluso en diferente fecha, así como también, favorece la conservación del semen por periodos de tiempo indefinidos $(2,3)$.

La población canina en Colombia ha tenido un crecimiento anual del 5\%, de igual forma, en la ciudad de Medellín el crecimiento durante el mismo periodo ha sido en promedio del 14\%. Por esta razón el uso de herramientas biotecnológicas tales como la inseminación artificial, así como la criopreservación de semen canino, podría potencializar el proceso productivo y reproductivo en el mercado canino de la ciudad y del país. Sin embargo, la aplicación y desarrollo de estas herramientas ha sido baja a nivel regional (4).

Por otra parte, el tipo de manejo y alimentación de los caninos y las condiciones ambientales propias del valle del aburra, en donde se encuentra ubicada la ciudad de Medellín, podrían influir en las características seminales de los perros usados para la reproducción, al ser comparados con las características reportadas por otros autores en diferentes países. Por lo anterior, en este trabajo se buscó aplicar la metodología de criopreservación rápida, mediante el uso pajilla francesa en semen canino en el municipio de Medellín- Antioquia, en la búsqueda de contribuir con el desarrollo de las biotecnologías reproductivas para esta especie.

\section{MATERIALES Y MÉTODOS}

Muestra. Se colectaron ocho caninos de las razas: bulldog francés, doberman, pastor alemán, Border collie y Boston terrier entre los 2 y 5 años, que se encontraron sanos en el examen clínico.

Colecta de semen. Se realizó en 8 caninos, previo a las colectas se les concedió un periodo de descanso sexual de 6 días, el método utilizado para la colecta fue el método de mano enguantada descrito por Olivera y Gobelo (2), se colectó solamente la fracción espermática, la cual se depositó en un frasco citoquímico estéril a temperatura entre 35 a $37^{\circ} \mathrm{C}(2,4)$. 
Evaluación postcolecta. Cada una de las muestras obtenidas fue evaluada para determinar las características seminales macroscópicas y microscópicas.

Evaluación macroscópica. Apariencia: Se evaluó visualmente el aspecto y color del semen. Volumen: Se evaluó directamente, la muestra del semen se pasó desde el frasco citoquímico donde se encontraba almacenada a un tubo cónico falcón (BD, Estados Unidos) de 15 cm y en este tubo se realizó directamente la medición del volumen.

Evaluación microscópica. Concentración de espermatozoides: Se realizó una dilución de 1:200 de una muestra de $5 \mu \mathrm{L}$ de semen con agua destilada y posteriormente bajo visión de microscopio (Olympus corporation, Tokio, Japón) a 40X se evaluó por medio de la cámara de Neubauer (Marienfeld, lauda-königshofen, Alemania) de acuerdo con lo reportado por Watts (5). Motilidad Individual: Se extrajo una gota de semen y se puso sobre un portaobjetos, se evalúo al microscopio con un objetivo de $40 X(5,6)$. Se determinó el porcentaje de espermatozoides en movimiento y la calidad según el tipo de movimiento (progresión lineal, progresión no lineal, no progresiva e inmóvil). El vigor, fue evaluado junto con la motilidad, teniendo en cuenta para su evaluación la velocidad con la que los espermatozoides atravesaron el campo. Se clasificó de acuerdo con lo descrito por Restrepo et al (6) de 1-5 (1 malo 5 excelente).

\section{Procesamiento de muestras}

Dilución. Para la dilución del semen se utilizó el diluyente comercial Triladyl ${ }^{\circledR}$; este fue seleccionado teniendo en cuenta los buenos resultados presentados en la dilución del semen bovino en otros estudios $(7,8,9,10)$, además se utilizó por ser un diluyente de fácil adquisición en el mercado y ser económico comparado con los diluyentes comerciales diseñados para caninos.

Se extrajo un pequeño volumen de semen para realizar la evaluación de las características del semen fresco y posteriormente se procedió a realizar una predilución de protección del semen en relación 1:1 con el diluyente para ser transportado posteriormente al laboratorio. Tras la llegada al laboratorio, se evaluó la motilidad individual y el vigor del semen nuevamente para luego llevarlo a una concentración de 80 millones de espermatozoides por ml.

Empaque y sellado. Para el empacado del semen se utilizaron pajillas francesas de 0.5 ml, estas fueron selladas con polivinilo utilizando un equipo procesador de semen (Minitube, Tiefenbach, Alemania).

Curva de descenso de temperatura. Las pajillas fueron llevadas a refrigeración en una nevera, esta se realizó desde $24^{\circ} \mathrm{C}$ (temperatura de laboratorio) hasta $4^{\circ} \mathrm{C}$, permitiendo así, que el diluyente interactuara con el semen, también para que se redujera el metabolismo celular y evitar la toxicidad del agente crioprotector que contiene el diluyente; este descenso de temperatura se realizó durante 3-4 horas (6).

Congelación: Para el proceso de criopreservación por congelación rápida, las pajillas fueron sometidas a vapores de nitrógeno líquido $\left(\mathrm{N}_{2} \mathrm{~L}\right)$ en posición vertical durante 25 minutos a una distancia aproximada de $5 \mathrm{~cm}$ del nitrógeno. Posteriormente se bañaron con $\mathrm{N}_{2} \mathrm{~L}$ durante $15 \mathrm{~min}$, luego de este procedimiento se pasaron al termo de almacenamiento a una temperatura de $-196^{\circ} \mathrm{C}$ (temperatura del nitrógeno líquido) donde permanecieron almacenadas para su posterior evaluación.

Muestra de pajillas para evaluación. El tamaño de la muestra se tomó por método estadístico y completamente al azar, con un intervalo de confianza del $95 \%$.

Descongelación. Cada una de las pajillas se sumergió en agua a baño maría a temperatura de $37^{\circ} \mathrm{C}$ durante un minuto. Posteriormente se secaron con toallas de papel y se cortaron por el extremo sellado, finalmente el contenido seminal fue depositado en un tubo cónico (eppendorf) de $1.5 \mathrm{ml}$ previamente precalentado a $35^{\circ} \mathrm{C}$ aproximadamente.

Evaluación postdescongelación. Se depositó una gota de $30 \mu \mathrm{l}$ en un portaobjetos atemperado a $37^{\circ} \mathrm{C}$ posteriormente se cubrió con una laminilla y se evaluó en el microscopio (Olympus corporation) las variables motilidad y vigor de la misma forma que se realizó con el semen fresco $(7,11)$.

Análisis estadístico. Los datos de cada variable del semen fresco y postdescongelado se analizaron por método estadístico descriptivo; además de esto para las variables motilidad individual y vigor también se realizó un análisis de varianza (ANOVA) con una significancia de p<0.05 y una comparación de medias por test de Tukey para determinar la magnitud de la diferencia entre los datos de estas variables en el semen fresco y postdescongelado. 
Este estudio fue aprobado por el comité de ética de la Universidad de Antioquia para la experimentación con animales (Acta $\mathrm{N}^{\circ} 90$ del 22 de agosto, 2014, Medellín). Este estudio descriptivo se realizó en la ciudad de Medellín ubicada en la región del Valle de Aburrá, en la cordillera central de los Andes. A $1380 \mathrm{msnm}$, con una temperatura de $22^{\circ} \mathrm{C}$ en promedio.

\section{RESULTADOS}

De los ocho animales colectados por medio de la técnica de estimulación manual, se obtuvo un total de 8 eyaculados, los cuales fueron procesados, encontrándose los siguientes resultados:

Evaluación del semen fresco. En el 100\% de las colectas se evidenció una apariencia de semen blanquecino lechoso, el volumen promedio para los eyaculados fue de $2.27 \pm 1.42 \mathrm{ml}$, la concentración promedio fue de $388.5 \times 10^{6} \pm 228.069$ espermatozoides $/ \mathrm{ml}$; los valores obtenidos para las variables microscópicas motilidad individual y vigor fueron del $79 \pm 4 \%$ y $3.96 \pm 0.327$ respectivamente (Tabla 1 ).

Tabla 1. Resultado de las variables del semen fresco

\begin{tabular}{cccc}
\hline & \multicolumn{2}{c}{ Precongelación } & Desviación Estándar \\
\hline Variable & Promedio & \pm 1.40 \\
\hline Volumen & 2.27 & \pm 225.63 & - \\
Concentración & 388.50 & $\pm 4 \%$ & 0.0016 \\
Motilidad individual & $79 \%$ & \pm 0.323 \\
Vigor & 3.96 & 0.105 & \\
\hline
\end{tabular}

Parámetros evaluados postdescongelación. En total se obtuvieron 123 pajillas de los 8 eyaculados, de las cuales se

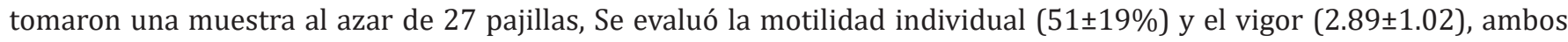
parámetros mostraron alta variabilidad entre los caninos. En la tabla 2 se pueden observar los resultados para cada variable postdescongelación.

Tabla 2. Resultados de las variables postdescongelación del semen

\begin{tabular}{cccc}
\hline & Postdescongelación & \\
\hline Variable & Promedio & Desviación Estándar & Varianza \\
\hline Motilidad individual & $51 \%$ & $\pm 19 \%$ & \pm 1 \\
Vigor & 2.89 & 1.016 & \pm 16 \\
\hline
\end{tabular}

Comparación entre la calidad del semen fresco y postdescongelado. En el análisis de varianza se encontró una significancia $(\mathrm{p}<0.05)$ entre las variables motilidad y vigor del semen fresco y postdescongelado. Al comparar la motilidad del semen fresco $(79 \pm 4 \%)$ con la del postdescongelado $(51 \pm 19 \%)$ se observa una diferencia significativa siendo mayor la del semen fresco (Tabla 1); de la misma forma el vigor del semen fresco (3.96 \pm 0.323$)$ es mayor comparado con el del semen postdescongelado $(2.89 \pm 1)$.

\section{DISCUSIÓN}

El proceso de colecta de semen utilizando la metodología de mano enguantada (2) logró la obtención del semen a partir del estímulo manual en todos los individuos, adicionalmente en todas las colectas se evidenció una apariencia del semen blanquecino lechoso, esto concuerda con lo reportado por Olivera y Gobello (2), Robert et al (4), Zorinkimi et al (12), Zorinkimi et al (13) y Shalini et al (14).

Con respecto al volumen del semen fresco se obtuvo un promedio de $2.27 \pm 1.4 \mathrm{ml}$ colectando solo la fracción espermática de los eyaculados, este valor es similar a lo descrito por Robert et al (4) quien informó que el volumen de la segunda fracción seminal está dentro del rango de 0,5 - $3.5 \mathrm{ml}$. Dobrinski et al (15) registró un volumen de $2.2 \pm 02 \mathrm{ml}$ para esta misma fracción en diferentes razas de perros y Olivera y Gobelo (2) reportaron un volumen de 0.1-6 ml. Los dos primeros autores indicaron que el volumen puede tener variaciones debido a las diferencias entre razas, lo anterior se evidenció en este estudio con una desviación estándar para el volumen de $\pm 1.40 \mathrm{ml}$. 
La concentración espermática promedio del semen fresco registrada en este estudio fue de $388.50 \times 10^{6} \pm 226$ espermatozoides por $\mathrm{ml}$, cifra que es superior a lo reportado por Nair et al (16) quienes registraron una concentración de $313 \times 10^{6} \pm 30.74$ espermatozoides/ml. Según Rota et al (17), Feldman et al (1), Morselli et al (7) y Sánchez (18) esta concentración espermática se encuentra dentro del rango normal para perros adultos. Sánchez (18) reportó una concentración espermática de $325 \times 10^{6} \pm 188$ espermatozoides/ml. Es importante destacar que según Feldman et al (1) un perro de tamaño medio que presente una concentración menor a 200×106 espermatozoides/ml viables postdescongelación es sospechoso de problemas de fertilidad, en este estudio ningún eyaculado presentó una concentración menor a la citada anteriormente.

Para las variables microscópicas del semen fresco se obtuvo que la motilidad espermática promedio fue de $79 \pm 4 \%$, destacando que en todos los eyaculados la motilidad progresiva fue superior al 70\%, el cual es el valor mínimo considerado para dar una buena calificación reproductiva en perros (1). Los valores de motilidad espermática encontrados en el 66\% de las muestras evaluadas fueron similares a los descritos por otros autores $(18,19,20)$, quienes señalan porcentajes de motilidad entre 80 y 90\%; Sin embargo, en este estudio se reportan motilidades menores al 79\% en varias de las razas de perros, con una motilidad en promedio del $75 \%$.

Por otra parte, cuando se evaluó el vigor, el valor promedio obtenido fue de $3.96 \pm 0.32$ en una escala de 1 a 5 , este parámetro indicó una muy buena movilidad en los espermatozoides de cada uno de los eyaculados evaluados, parámetro que podría asociarse con la capacidad de los espermatozoides para llegar al sitio de la fecundación (región itsmo-ampular del oviducto) (21).

Los valores obtenidos para la variable motilidad postdescongelación $(51 \pm 19 \%)$, según la prueba de Tukey difieren significativamente $(\mathrm{p}<0.05)$ de los valores obtenidos en el semen fresco $(79 \pm 4 \%)$. Este valor postdescongelación fue

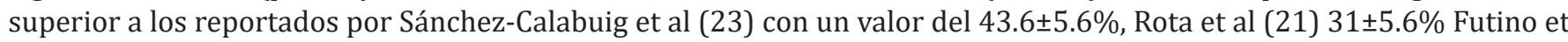
al (22) $37 \pm 19.8 \%$, y Restrepo et al (24) $44.5 \pm 17.7 \%$.

El vigor postdescongelación encontrado fue del 2.89 \$1.02 (en una escala de 1 a 5), evidenciándose una reducción con respecto al vigor del semen fresco. Un vigor inferior a 3, podría afectar la capacidad fecundante del semen (21), no obstante, en el 66.66\% de las muestras se obtuvieron vigores iguales o superiores a 3.

La reducción de los valores de las variables motilidad individual y vigor postdescongelación se podrían explicar por las alteraciones funcionales generadas en los espermatozoides debido a los cambios de temperatura (shock de frío), la toxicidad del crioprotector, la formación y disolución de hielos en el medio ambiente extracelular (3, 6, 24) , el estrés osmótico al que se ven sometidos los espermatozoides en la criopreservación, los daños que sufren a nivel estructural, las modificaciones en el metabolismo celular y los daños en la membrana espermática $(22,23)$, con relación a lo anterior Martínez et al (25) propone que los daños en la mitocondria como la fragmentación de su ADN pueden ser un factor relevante en la disminución de la motilidad espermática, pues es la mitocondria quien codifica las proteínas necesarias para la respiración celular aerobia, la cual tiene como objetivo la producción de ATP, molécula que brinda la energía para la motilidad espermática. Respecto a lo anterior, Restrepo et al (24) proponen que la exagerada formación de especies reactivas de oxígeno por los daños de membrana causados por el diluyente y la refrigeración son los factores directamente involucrados en las alteraciones anteriormente mencionadas.

A pesar de los daños que pueden sufrir los espermatozoides por el proceso de criopreservación, en este estudio la motilidad y el vigor de estos, aunque mostraron una diferencia significativa $(\mathrm{p}<0.05)$ con respecto al semen fresco, son valores que continúan siendo aptos para el proceso de inseminación artificial.

En conclusión, el método de mano enguantada utilizado para la estimulación y colecta de los caninos permite la obtención de semen con buenas características para ser evaluado, diluido y posteriormente congelado.

La dilución del semen canino con el diluyente comercial Triladyl ${ }^{\circledR}$ diseñado para uso en bovinos, mostró tener resultados satisfactorios en la criopreservación de semen canino, puesto que a pesar de que el proceso de congelación causó una disminución en los parámetros de motilidad y vigor, estas características seminales teniendo en cuenta diferentes autores continúan estando dentro de los rangos normales para el semen postdescongelado.

El proceso de criopreservación seminal puede causar daños en la membrana citoplasmática, alterar el metabolismo espermático y afectar los parámetros de evaluación seminal como la motilidad individual y el vigor postdescongelación al ser estas variables comparadas con el semen fresco. 
La aplicación del método de pajilla francesa para la criopreservación de semen canino demostró ser efectivo; obteniéndose valores postdescongelación aceptables en las variables motilidad individual y vigor, lo cual puede permitir su utilización en procesos de inseminación artificial.

Se recomienda realizar futuras investigaciones encaminadas a evaluar la eficiencia de la metodología en otras variables como la viabilidad, la morfología de los espermatozoides, la funcionalidad de la membrana plasmática y la actividad mitocondrial, para poder evidenciar resultados con mayor confianza.

Se sugiere realizar diferentes investigaciones orientadas a mejorar las diferentes características fecundantes del semen canino postdescongelación, buscando la prevención y disminución de los eventos que generan daños funcionales y de membrana.

\section{Conflicto de intereses}

Los autores declaramos no tener conflictos de interés con respecto al trabajo aquí publicado

\section{Agradecimientos}

Agradecemos a los docentes Andrés Mejía Tamayo y Carlos Javier Tabares por su colaboración en la ejecución de esta investigación. También agradecemos a la Unidad de Biotecnología Reproductiva de la Universidad de Antioquia por la financiación parcial del proyecto.

\section{REFERENCIAS}

1. Feldman, E.; Nelson, R. Canine and feline endocrinology and reproduction. Tercera edición; 2003. Editorial Saunders Co. Philadelphia, USA. ISBN: 9781416064572. URL Disponible en: https://www.elsevier.com/books/canine-andfeline-endocrinology-and-reprod/feldman/978-0-7216-9315-6

2. Olivera M, Gobello C. El libro latinoaméricano de reproducción canina y felina. Medellín, Colombia: Fondo editorial biogénesis; 2005. URL Disponible en: https://edicioneselprofesional.com.co/es/tienda/el-libro-latinoamericanode-reproduccion-canina-y-felina

3. Stornelli MA, Stornelli MC, Arauz L. Inseminación artificial con semen fresco, refrigerado y congelado. Aplicación y desarrollo en caninos. Analecta Veterinaria 2001; 21(1):58-66. URL Disponible en: http://www.fcv.unlp.edu.ar/ images/stories/analecta/vol $21 \mathrm{n} 1 / 056$ VE21n1 stornelli inseminacion caninos.pdf

4. Robert MA, Jayaprakash G, Pawshe M, Tamilmani T, Sathiyabarathi M. Collection and evaluation of canine semen-a review. International Journal of Science, Environment and Technology. 2016; 5(3):1586-1595. http://www.ijset. net/journal/1040.pdf

5. Watts J. Development of a fluorescent computer-assisted spermatozoal quantification method and a comparison of results for manual counting with a haemocytometer and computer-assisted semen analysis in dogs. Reprod Domest Anim. 2019; 54(11):1477-1488. https://doi.org/10.1111/rda.13553

6. Restrepo G, Vasquez N, Garcia E. 9. Criopreservacion de Semen canino y su aplicación en la Inseminación artificial. Rev CES Med Zootec. 2009. 4(2):119-129. URL Disponible en: http://revistas.ces.edu.co/index.php/mvz/article/ view/1038/1910

7. Morselli MG, Colombo M, Faustini M, Luvoni GC. Morphological indices for canine spermatozoa based on the World Health Organization laboratory manual for human semen. Reprod Domest Anim. 2019; 54(7):949-955. https://doi. org/10.1111/rda.13440

8. Kang S-S, Lee M-S, Kim U-H, Lee S-D, Yang B-C, Yang B-S, et al. Effect of Optixcell and Triladyl extenders on frozenthawed sperm motilities and calving rates following artificial insemination in Hanwoo. Korean Journal of Agricultural Science. 2019; 46(1):195-204. https://doi.org/10.7744/kjoas.20190009

9. Miguel-Jimenez S, Rivera del Alamo MM, Álvarez-Rodríguez M, Hidalgo CO, Peña AI, Muiño R, et al. In vitro assessment of egg yolk-, soya bean lecithin- and liposome-based extenders for cryopreservation of dairy bull semen. Anim Reprod Sci. 2020; 215:106315. https://doi.org/10.1016/j.anireprosci.2020.106315 
10. Argudo DE, Galarza DA, Bueno P, Iñiguez CU, Méndez S, Soria ME, et al. Methods of collection, extender type, and freezability of semen collected from creole bulls raised in the tropical highlands of Ecuador. Trop Anim Health Prod. 2019; 51(7):1839-1845. https://doi.org/10.1007/s11250-019-01877-3

11. Buranaamnuay K. Protocols for sperm cryopreservation in the domestic cat: A review. Anim Reprod Sci. 2017; 183:56-65. https://doi.org/10.1016/j.anireprosci.2017.06.002

12. Zorinkimi A, Ahmed FA, Lalrintluanga K, Kuotsu N. Characterization of mongrel dog semen of mizoram. Int J Curr Microbiol Appl Sci. 2017; 6(12):1140-1145. https://doi.org/10.20546/ijcmas.2017.612.128

13. Zorinkimi A, Ahmed FA, Lalrintluanga K. Effect of different extenders on the quality of mongrel dog semen preserved at $5^{\circ} \mathrm{C}$ on the basis of hypo-osmotic sperm swelling test (HOSST). Int J Curr Microbiol Appl Sci. 2017; 6(12):961-964. https://doi.org/10.20546/ijcmas.2017.612.106

14. Shalini I, Antoine D. Semen characteristics in german shepherd dogs. Int J Curr Microbiol Appl Sci. 2018; 7(3):23042312. https://doi.org/10.20546/ijcmas.2018.703.270

15. Dobrinski I, Lulai C, Barth AD, Post K. Effects of four different extenders and three different freezing rates on post-thaw viability of dog semen. J Reprod Fertil Suppl. 1993; 47:291-296. https://www.ncbi.nlm.nih.gov/pubmed/8229939

16. Nair Srm, Kalatharan J, Rajasekaran J. Effect of cryopreservation on the viability and membrane integrity of canine spermatozoa. Indian J Anim Reprod. 1999; 20:142-145.

17. Rota A, Rota A, Martini M, Milani C, Romagnoli S. Evaluation of dog semen quality after slow (biological freezer) or rapid (nitrogen vapours) freezing. Reprod Nutr Dev. 2005; 45(1):29-37. https://doi.org/10.1051/rnd:2005002

18. Sánchez A, Rubilar J, Gatica R. Uso de la prueba hipoosmótica en la evaluación de la fertilidad potencial de semen canino fresco y congelado. Arch Med Vet. 2002; 34(1). http://dx.doi.org/10.4067/S0301-732X2002000100014.

19. Pan $\mathrm{C}, \mathrm{Wu}$ Y, Yang Q, Ye J. Effects of seminal plasma concentration on sperm motility and plasma and acrosome membrane integrity in chilled canine spermatozoa. Pol J Vet Sci. 2018; 21(1):133-138. DOI https://doi. org/10.24425/119031

20. Lucio C, Brito M, Angrimani D, Belaz K, Morais D, Zampieri D, et al. Lipid composition of the canine sperm plasma membrane as markers of sperm motility. Reprod Domest Anim. 2017; 52:208-213. https://doi.org/10.1111/ $\underline{\text { rda. } 12860}$

21. Rota A, Milani C, Cabianca G, Martini M. Comparison between glycerol and ethylene glycol for dog semen cryopreservation. Theriogenology. 2006; 65(9):1848-1858. https://doi.org/10.1016/j.theriogenology.2005.10.015

22. Futino D, Mendes M, Matos W, Mondadori R, Lucci C. Glycerol, Methyl-Formamide and Dimethyl-Formamide in Canine Semen Cryopreservation. Reprod Domest Anim. 2010; 45(2):214-220. https://doi.org/10.1111/j.1439$\underline{0531.2008 .01208 . x}$

23. Sánchez-Calabuig MJ, Maillo V, Beltrán-Breña P, de la Fuente Martínez J, Galera-Carrillo S, Pérez-Gutiérrez JF, et al. Cryopreservation of canine sperm using egg yolk and soy bean based extenders. Reprod Biol. 2017; 17(3):233-238. https://doi.org/10.1016/j.repbio.2017.05.007

24. Restrepo Betancur Giovanni, Gómez Oquendo Jorge, Vásquez Araque Neil. Criopreservación de semen canino por congelación rápida con glicerol y Dimetilformamida. Rev Lasallista Investig; 2011; 8(2):9-17. URL Disponible from: http://repository.lasallista.edu.co:8080/ojs/index.php/rldi/article/view/19.

25. Martinez José G, Pardo C. Sandra. Crioconservación: efectos sobre la movilidad espermática y la fertilización en peces. Acta Biológica Colombiana. 2010; 15(2):3-24. https://revistas.unal.edu.co/index.php/actabiol/article/ view/10868/28159 